\title{
LDPC-based Joint Source Channel Coding and Decoding Strategies for single relay cooperative communications
}

\author{
Marwa Ben Abdessalem*, Amin Zribi*†, Tadashi Matsumoto ${ }^{\ddagger}$, Elsa Dupraz ${ }^{\dagger}$, and Ammar Bouallègue* \\ *University of Tunis El Manar, National Engineering School of Tunis, SysCom laboratory, BP 37, Le Belvedere, 1002, Tunis \\ Email: marwa.benabdessalem@enit.utm.tn, ammarbouallegue.syscom@gmail.com \\ $\dagger$ IMT Atlantique, Lab-STICC, UBL, 29238 Brest, France \\ Email: amin.zribi@isetcom.tn, elsa.dupraz@imt-atlantique.fr \\ $\ddagger$ Japan Advanced Institute of Science and Technology,1-1 Asahidai,Nomi,Ishikawa, Japan, \\ and Centre for Wireless Communications (CWC), University of Oulu, Finland (Part-time) \\ Email:matumoto@jaist.ac.jp
}

\begin{abstract}
This paper investigates new cooperative communication strategies based on Low-Density Parity-Check codes (LDPC) with Joint Source-Channel (JSC) coding and decoding algorithms to efficiently deliver a correlated content to a destination node. The proposed strategies rely on double LDPC codes where the source and the channel coding are similar to standard LDPC operations, and can be mapped on Tanner graphs for message-passing decoding. Three coding strategies are proposed where different LDPC source and channel coding setups are applied at the source node and the relay node. For each transmission strategy, we propose a graph mapping the whole network, over which iterative message-passing decoding is applied, and where the probability of errors in the source-relay link are taken into account. We showed, based on computer simulations, that the proposed strategies can provide substantial improvements compared to equivalent rates point-to-point systems. Simulation results also showed that the system performance depends on the relay position, and that adapting the source and the relay channel coding rates accordingly can enhance the overall system performance for a time-varying network.
\end{abstract}

Keywords: Source Channel Coding Strategies, Joint Source Channel coding/decoding, LDPC codes, cooperative communication.

\section{INTRODUCTION}

For point-to-point digital communication systems, Shannon's separation theorem [1] states that reliable communication can be achieved while still retaining the system performance by separate source and channel coding. The separation theorem was demonstrated under the assumption of infinite information block lengths over stationary channels with arbitrary low error probability. However, with such idealized assumptions, it is typically difficult to implement the separation design for practical communication systems with complexity, delay, energy and bandwidth constraints.

Because of these practical limitations, Joint Source and Channel Coding (JSCC) designs were proposed a good alternative for reliable communication over noisy channels [2]-[4]. In several new decision-making communication systems, like Wireless Multimedia Sensor Networks (WMSN) [5] or Multimedia Internet of Things (MIoT) [6], the design goal changed and residual error rates are tolerated as long as the decision is reliable and the system is of low complexity and especially consuming low energy. During many years, authors considered entropy encoding techniques for the source coding part, followed by a Joint Source Channel (JSC) decoding process in order to exploit the remaining source redundancy after compression [7], [8]. In this framework, Variable Length Codes (VLC) and Arithmetic Codes (AC) were used since they allow to reduce the data redundancy and to approach the source entropy [9]-[11]. Unfortunately, VLC and AC are very sensitive to errors induced by the channel noise, which leads to error propagation, and to a higher source error rates. In addition, the entropy-approaching source codes are generally impractical, because they require the knowledge of the probability of each symbol, which increases the requirements of memory and complexity, especially when the symbols are chosen from an alphabet having a large cardinality. Other works, targeting wireless sensor networks applications, omitted source compression to reduce the encoder complexity and energy consumption. JSC decoding methods were proposed to exploit the source correlation at the receiver [12]. However, under hard bandwidth consumption constraints, transmitting uncompressed data is not reasonable. Based on the success of Low-Density Parity-Check codes (LDPC) as capacity approaching channel codes, Fresia et al. proposed [3] a JSC encoding scheme based on LDPC codes. The structure of the encoder is composed by a source LDPC code that compresses the data source, followed by a channel LDPC code that aims to provide a good transmission reliability. At the receiver, the double LDPC system, represented by a unique Tanner graph, allows iterative joint decoding based on the standard Belief Propagation (BP) algorithm. The double LDPC code can be a very good solution for the above mentioned WMSNs and MIoT applications 
because of its simplicity and low latency. Indeed, good performance improvements can be achieved compared to classical JSC solutions [3] when low error floors are tolerated even at high Signal-to-noise ratio (SNR).

On the other hand, relaying in wireless cooperative communication has attracted a lot of interest in recent years, since it affords diversity gain, increases the channel capacity, and lowers the power cost required to enhance the performance of the communication system. A simple cooperative system is composed of a single source, one destination, and one (or many) relay(s), where the relay helps the source node by forwarding new combinations of the source message to the destination. The relay can perform different operations, such as Amplify-and-forward (AF) [13], Decode-and-Forward (DF) [14], Compressand-Forward (CF) [15]. Among these different approaches, many DF relaying strategies were implemented using distributed Turbo Codes or LDPC codes. In [14], a distributed Turbo Code was proposed, under the assumption of having a reliable link between the source and the relay. In [16], authors extended the study to show that the use of Turbo Codes improves the performance of the cooperative networks, even when the source-relay link is not perfect. Moreover, authors in [17] investigated distributed protograph LDPC codes for the relay system to improve error correction capacity. Motivated by the benefits of LDPC codes [18], authors in [19] and [20] designed new LDPC-based communication strategies for the half-duplex relay channel. Also, authors in [21] designed the root-protograph (RP) LDPC codes for ergodic and non-ergodic block fading (BF) channels exploiting the modified protograph EXIT (PEXIT) algorithms. In [22], authors provide a comprehensive overview of the full diversity maximum-distance separable RP codes, based on code design through BF channels. In [23], authors considered LDPC codes with iterative decoding on block-fading relay channels, where two users communicate their own information using Coded Cooperation (CC). Furthermore, an optimization method of irregular LDPC codes for the half duplex relay channels using the DF strategy was proposed in [24], based on Density Evolution (DE). A relay selection algorithm combined with joint network coding was proposed in [25] to improve the performance of a Two-Way Relay Network (TWRC) based on differential chaos shift keying (DCSK). The above mentioned Turbo and LDPC cooperative communication solutions were designed under the assumption of a non correlated source, which is not always the case especially for multimedia communications. For such applications, many authors investigated the cooperative communication system design, involving source coding with the target to exploit the network spatial correlation and the data inherent temporal correlation. In [26], a distributed JSCC scheme was proposed to transmit Markov sources, exploiting the temporal correlation based on a modified Bahl-Cocke-Jelinek-Raviv (BCJR) algorithm and the spatial correlation of the relay system. Also, new source and channel coding cooperative schemes were proposed in [27]. The proposed solutions allow to exploit the correlation between sensor nodes and the wireless Additive White Gaussian Noise (AWGN) channel to facilitate the communication between different network components. Moreover, an iterative decoding algorithm that uses the correlation between the source and the relay nodes was proposed in [28] based on an accumulator-assisted distributed Turbo Code. Even efficient, we note that all the above mentioned contributions are not using source compression, which induces high bandwidth and high energy-consuming transmission.

In this paper, we investigate the transmission of correlated sources through noisy channels with the help of a relay, based on the JSCC double LDPC codes. In this context, the main question we want to address is how to distribute the source compression and the channel coding operations between the source and the relay nodes. To answer this question, we propose three different Source Channel Coding Strategies (SCCSs). We show that each coding/communication strategy can be mapped on a specific Tanner graph with a corresponding message-passing decoding algorithm that aims at exploiting both the residual redundancy of the source and the network correlation to estimate the original information. The main contributions of this paper are: 1) We propose different SCCSs with distributed source compression and channel coding operations based on JSCC LDPC applied to a cooperative setup. 2) We designed a joint decoding process for each proposed strategy, based on modified BP iterative algorithms and taking into account the case where errors occur in the source-relay link. 3) We evaluate the performance of each SCCS with different relay positions and for different error-probabilities between the source and the relay nodes. This analysis allows to select the best strategy depending on the source, the channel, and the relaying conditions.

The remainder of this article is organized as follows. In Section II, we present the background of this work, and we define the treated problem with the corresponding notations. The proposed iterative JSC decoding methods and strategies are detailed in Section III. Simulation results and discussions are depicted in Section IV. Finally, conclusions are given in Section V.

\section{Context And PRoblem Statement}

\section{A. LDPC codes for JSC coding and decoding}

The standard LDPC JSCC scheme uses two concatenated LDPC codes respectively employed for source compression and the channel coding. The receiver performs joint decoding of the source and the channel LDPC codes [2], [3] using the Belief Propagation (BP) algorithm. The JSCC structure is described in more details as follows.

The LDPC source compression operation aims at reducing the redundancy of the original source using fixed-to-fixed length coding. All the compressed bit-streams have the same length, which is useful for the JSC decoding as it avoids catastrophic 
affection of the decoded source information after residual transmission errors. We consider a data source and a $(l \times n)$ parity check matrix of the source code $\mathbf{H}_{s c}$. Applying this parity check matrix for encoding, we can compress the source as

$$
\mathbf{b}=\mathbf{H}_{s c} \times \mathbf{s},
$$

where $\mathbf{s}$ is a vector indicating the $n$-source symbols length and $\mathbf{b}$ is the compressed sequence with $l<n$ bits.

The LDPC channel coding operation is used to combat the channel imperfections by adding redundancy to the source compressed as:

$$
\mathbf{c}=\mathbf{G}_{c c}^{T} \times \mathbf{b}=\mathbf{G}_{c c}^{T} \times \mathbf{H}_{s c} \times \mathbf{s},
$$

where $\mathbf{c}$ is the $m$-bits codeword, and $\mathbf{G}_{c c}$ is the $(l \times m)$ generator matrix. We define the $R_{s c}=\frac{l}{n}$ as the source coding rate, and $R_{c c}=\frac{l}{m}$ as the channel coding rate. The overall rate of the system is $R=\frac{n}{m}$.

The LDPC-based JSC message-passing decoding is performed on a Tanner graph with the BP algorithm by exchanging extrinsic log-likelihood ratios (LLRs) between the source and the channel LDPC decoders. In general, the Tanner graph of the channel code, as well as of the source code are described by two sets of nodes: the variable nodes and the check nodes representing respectively the columns and the rows of their parity check matrices.

The JSCC LDPC flexibility and performance motivated many recent works [29]-[35]. In [29], authors considered double photograph-based JSCC LDPC codes for radiography image transmission, and provided channel SNR (Signal-to-Noise Ratio) improvements. In another context, a coding and decoding scheme based on Joint Source-Channel-Network (JSCN) LDPC coding was proposed in [31] for the Multiple Access Relay Channel (MARC), and demonstrated good BER performance improvements. The state-of the art of the applications enabled by protograph LDPC codes are studied in [32] including joint channel-and-physical-layer network coding. Many recent works, motivated by the fact that the JSCC LDPC codes distortion and correction levels are related to the code construction, provided optimized protograph construction for both the channel and the source LDPC codes to lower the decoding error floor [33], [34]. In a very recent work [35], authors proposed a joint coding scheme for transmitting images based on DP-LDPC especially designed for for IoT applications. To further enhance the system reliability authors proposed an improved rate allocation strategy based on different combinations of optimized LDPC code pairs.

\section{B. Problem statement}

As aforementioned, we study a cooperative communication setup composed of one source, single relay, and one destination with two transmission phases, where the source node communicates its own correlated data to a destination with the help of a relay. Besides, we focus on JSC coding and decoding solutions based on LDPC codes for cooperating nodes. Indeed, our objective is to determine how to better apply the JSCC LDPC codes in a cooperative system, depending on source and channel coding. Hence, we propose three Source Channel Coding Strategies (SCCS1, SCCS2, SCCS3) for source and channel coding in a cooperative system, with the purpose to study how to distribute the source compression and the channel coding operations between the source and the relay nodes. For the proposed SCCSs, we consider the transmission of binary Markov sources and keep the same overall transmission rate for a fair comparison. In general, a Markov source $\left(S_{t}\right)$ is modeled by two transition probabilities: $\alpha=\operatorname{Pr}\left(S_{t}=1 \mid S_{t-1}=0\right)$ and $\beta=\operatorname{Pr}\left(S_{t}=0 \mid S_{t-1}=1\right)$. The binary entropy is denoted by $h(x)=-x \log _{2}(x)-(1-x) \log _{2}(1-x)$. The entropy of the Markov source is calculated as $H(S)=\mu_{0} h(\alpha)+\mu_{1} h(\beta)$, where $\mu_{0}=1-\mu_{1}=\frac{\beta}{\beta+\alpha}$ is the bit 0 stationary probability distribution. In addition, for each SCCS, we recall that the wireless source-relay link is supposed to be noisy, and the reconstructed sequence $\hat{\mathbf{x}}$ at the relay node can be an erroneous version of $\mathbf{x}$ corrupted with a bit error probability $p_{e}$. Therefore, the corresponding joint decoder should take into consideration this error probability to estimate the sequence sent by the source node. This can be done by applying an updating function $f_{c}$ based on the appropriate LLRs of $\hat{\mathbf{x}}$ and the error probability $p_{e}$. The function $f_{c}$ is defined as follows [26]

$$
\begin{aligned}
\operatorname{LLR}(x) & =\log \left(\frac{\exp (\operatorname{LLR}(\hat{x}))\left(1-p_{e}\right)+p_{e}}{\left(1-p_{e}\right)+\exp (\operatorname{LLR}(\hat{x})) p_{e}}\right) \\
& =f_{c}\left(\operatorname{LLR}(\hat{x}), p_{e}\right)
\end{aligned}
$$

In the next Section, we describe the three possible encoding strategies (SCCSs) with their corresponding decoding methods, based on the iterative message-passing algorithm, and the LLRs updating applied to the network factor graph.

\section{Notations}

In the following, we introduce the notations used in this article:

- $\mathcal{V}_{c c}$ and $\mathcal{V}_{s c}$ are, respectively, the sets of variable nodes of the LDPC channel decoder graph and the LDPC source decoder graph.

- $\mathcal{C}_{c c}$ and $\mathcal{C}_{s c}$ are, respectively, the sets of check nodes of the LDPC channel decoder graph and the LDPC source decoder graph. 


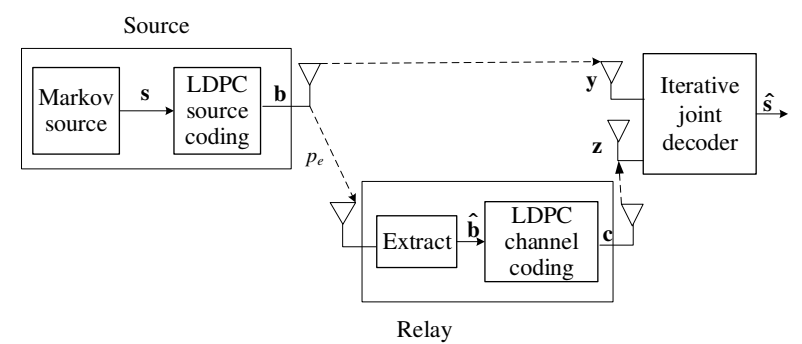

(a) System model for Source Channel Coding Strategy 1 (SCCS1)

Fig. 1: The SCCS1 for the cooperative system and the corresponding Joint Tanner graph decoder, exploiting source-relay correlation.

- $\mathcal{V}_{c c}^{s y s t}$ and $\mathcal{V}_{c c}^{r e d}$ are the sets of variable nodes corresponding, respectively to the systematic bits and to the parity bits of the LDPC channel decoder graph.

- $m_{i, j}^{c c}$ and $m_{i, j}^{s c}$ are respectively the messages sent from the node $i \in\{v, c\}$ to the node $j \in\{c, v\}$ of the LDPC channel decoder graph and the LDPC source decoder graph, where $v$ and $c$ denote the variable and check nodes, respectively.

- $Z_{v}^{s c}$ and $Z_{v}^{c c}$ are, respectively the initial intrinsic channel LLRs of the variable nodes of the LDPC source decoder graph and the LDPC channel decoder graph.

- $Z_{c}^{s c}$ denote the initial channel LLRs of the check nodes of the LDPC source decoder graph.

In the proposed decoding algorithms, we also consider information transfer between the graphs of the network component codes. The messages transferred from a graph $i \in\{s c, c c\}$ to a graph $j \in\{c c, s c\}$ are denoted $m_{v}^{i \rightarrow j}$.

\section{LDPC SOURCE ChANNEL CODING STRATEGIES FOR COOPERATIVE COMMUNICATION}

\section{A. SCC Strategy 1 (SCCS1)}

In the proposed SCC Strategy 1, the JSCC LDPC compression and error protection operations are distributed at the source and the relay nodes respectively. There are two transmission phases. In the first phase, as shown in Fig. 1 (a), the source generates correlated data $\mathbf{s}$ with $n$ symbols. The latter is compressed by an LDPC source encoder as specified by the equation (1). The compressed sequence $\mathbf{b}$ is transmitted to both the relay node and the destination node.

In the second phase, the relay extracts the compressed sequence, by applying hard binary decision. The obtained sequence $\hat{\mathbf{b}}$ is then encoded by an LDPC channel code as

$$
\mathbf{c}=\mathbf{G}_{c c}^{T} \times \hat{\mathbf{b}}
$$

where the codeword $\mathbf{c}$ is modulated and transmitted to the destination over an AWGN channel. After receiving the signals from the source and the relay, the destination retrieves the original information using joint-based LDPC decoder. The overall rate of the proposed system is $R=\frac{n}{(l+m)}$, where $R_{s c}=\frac{l}{n}$ is the compression rate and $R_{c c}=\frac{l}{m}$ is the channel coding rate.

At the receiver, we propose a joint decoder that uses message transfer based on the standard BP algorithm applied on a two concatenated Tanner graphs representing the coding system shown in Fig. 1 (b). In this decoder, the LLRs sent from $v \in \mathcal{V}_{s c}$ to $c \in \mathcal{C}_{s c}$ are

$$
m_{v, c}^{s c}=Z_{v}^{s c}+\sum_{c^{\prime} \neq c} m_{c^{\prime}, v}^{s c}
$$

Since we consider symmetric Markov source $\left(\mu_{0}=\mu_{1}\right)$, the initial LLRs are set to $Z_{v}^{s c}=0$. Then, for all $v \in \mathcal{V}_{c c}^{\text {syst }}$, the messages passed to check nodes $c$ are evaluated as

$$
m_{v, c}^{c c}=Z_{v}^{c c}+f_{c}\left(m_{v}^{s c \rightarrow c c}, p_{e}\right)+\sum_{c^{\prime} \neq c} m_{c^{\prime}, v}^{c c}
$$

The initial channel LLRs are $Z_{v}^{c c}=\frac{2 r_{v}}{\sigma_{R D}^{2}}$, where $\sigma_{R D}^{2}$ is the $R$-D channel noise variance, and $r_{v}=\left(1-2 c_{v}\right)+n_{v}$ with $n_{v}$ is a random channel noise sample. $m_{v}^{s C B C c}$ represents the messages delivered by the check nodes of the source graph decoder to the variable nodes of the channel graph decoder. Also, assuming a noisy link between the source and the relay nodes, 


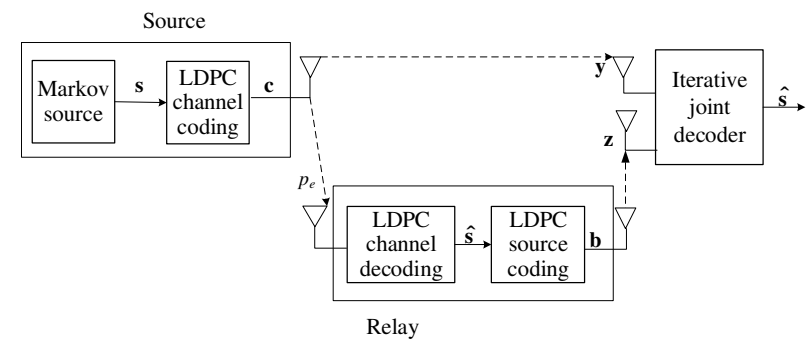

(a) System model for Source Channel Coding Strategy 2 (SCCS2)

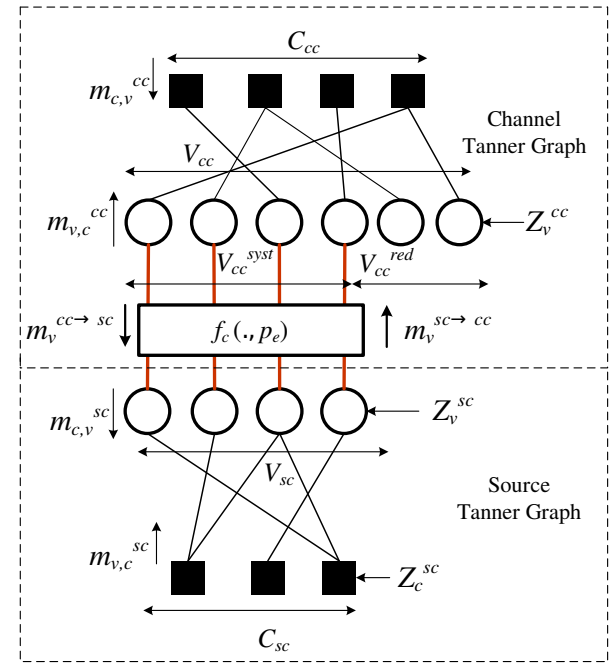

(b) Network Tanner graph and message transfer for SCCS2

Fig. 2: The SCCS2 for the cooperative system and the corresponding decoding graph, exploiting source-relay correlation.

we remind that the signal $\hat{\mathbf{b}}$ is corrupted with an error probability $p_{e}$. Hence, the joint Tanner graph estimates this signal by applying the $f_{c}$ function previously indicated according to equations (3) and (4). Then, for $v \in \mathcal{V}_{c c}^{s y s t}$, we have

$$
m_{v}^{c c \rightarrow s c}=Z_{v}^{c c}+\sum_{c^{\prime}} m_{c^{\prime}, v}^{c c}
$$

where $m_{v}^{c c \rightarrow s c}$ are the messages passed from the $v$ nodes of the channel Tanner graph to the $c$ nodes of the source Tanner graph. For $v \in \mathcal{V}_{c c}^{r e d}$, the messages transfer from the $v$ nodes to the $c$ nodes are

$$
m_{v, c}^{c c}=Z_{v}^{c c}+\sum_{c^{\prime} \neq c} m_{c^{\prime}, v}^{c c}
$$

We note that messages are initialized by $m_{c^{\prime}, v}^{c c}=0, m_{v}^{s c \rightarrow c c}=0$, and $m_{c^{\prime}, v}^{s c}=0$.

For the same iteration, every the check node $c$ responds with its LLRs to a connected variable node $v$. For $c \in \mathcal{C}_{s c}$, we have the following messages

$$
m_{c, v}^{s c}=2 \tanh ^{-1}\left(\tanh \left(\frac{Z_{c}^{s c}}{2}\right) \prod_{v^{\prime} \neq v} \tanh \left(\frac{m_{v^{\prime}, c}^{s c}}{2}\right) \times \tanh \left(\frac{f_{c}\left(m_{v}^{c c \rightarrow s c}, p_{e}\right)}{2}\right)\right)
$$

where $Z_{c}^{s c}=\frac{2 r_{c}}{\sigma_{S D}^{2}}$ represent the noisy version of the sequence $\mathbf{b}$, with the received sample $r_{c}=\left(1-2 b_{c}\right)+n_{c}$, and $\sigma_{S D}^{2}$ is the source-destination channel noise variance. In addition

$$
m_{v}^{s c \rightarrow c c}=2 \tanh ^{-1}\left(\tanh \left(\frac{Z_{c}^{s c}}{2}\right) \prod_{v^{\prime}} \tanh \left(\frac{m_{v^{\prime}, c}^{s c}}{2}\right)\right)
$$

For $c \in \mathcal{C}_{c c}$, each check node $c$ sends to every connected $v$ node a message defined by:

$$
m_{c, v}^{c c}=2 \tanh ^{-1}\left(\prod_{v^{\prime} \neq v} \tanh \left(\frac{m_{v^{\prime}, c}^{c c}}{2}\right)\right)
$$

After a given number of iterations of the described messages transfer of the joint decoder, we can estimate the original information for $v \in \mathcal{V}_{s c}$, based on

$$
\operatorname{LLR}(\mathbf{s})=Z_{v}^{s c}+\sum_{c} m_{c, v}^{s c}
$$

\section{B. SCC Strategy 2 (SCCS2)}

In reverse to SCC Strategy 1, we propose as a second strategy, to use an LDPC channel code at the source node, in order to improve the reliability of the source-relay link, and an LDPC source code that operates at the relay node. As depicted in Fig. 2 (a), in the first transmission period of the SCC Strategy 2, the Markov source generates correlated information $\mathbf{s}$, which is encoded by an LDPC channel code using $(n \times m)$ generator matrix $\mathbf{G}_{c c}$ : 


$$
\mathbf{c}=\mathbf{G}_{c c}^{T} \times \mathbf{s}
$$

Then, the encoded sequence c of length $m$ is transmitted to both the relay and the destination nodes. During the second period, the relay decodes the received message $\mathbf{c}$ to obtain $\hat{\mathbf{s}}$ with the $((m-n) \times m)$ parity check matrix $\mathbf{H}_{c c}$, since applying channel decoding provides better performance than the hard binary decision at the relay node. After that, the sequence $\hat{\mathbf{s}}$ is compressed by an LDPC source encoder to obtain a compressed data, denoted $\mathbf{b}$ and calculated as

$$
\mathbf{b}=\mathbf{H}_{s c} \times \hat{\mathbf{s}}
$$

The sequence b of length $l$ is modulated by BPSK (Binary-Phase Shift Keying), and then forwarded to the destination. At the receiver, we propose a joint decoding process to retrieve the source data. We keep the same overall rate of the system as SCC Strategy 1, where $R_{c c}=\frac{n}{m}$ and $R_{s c}=\frac{l}{n}$. For SCC Strategy 2, the JSC decoding based on LDPC codes can be represented by two Tanner graphs as shown in Fig. 2 (b). First, the variable nodes $v$ of the LDPC channel decoder inform the check nodes $c$ about their LLRs, and the variable nodes $v$ of the LDPC source decoder send the messages to the corresponding check nodes $c$. For $v \in \mathcal{V}_{c c}^{s y s t}$, the LLRs are

$$
m_{v, c}^{c c}=Z_{v}^{c c}+\sum_{c^{\prime} \neq c} m_{c^{\prime}, v}^{c c}+f_{c}\left(m_{v}^{s c \rightarrow c c}, p_{e}\right)
$$

where

$$
m_{v}^{s c \rightarrow c c}=Z_{v}^{s c}+\sum_{c^{\prime}} m_{c^{\prime}, v}^{s c}
$$

are the messages passed from the variable nodes of the source Tanner graph to the variable nodes of the channel Tanner graph. Initial source and channel LLRs are evaluated as $Z_{v}^{c c}=\frac{2 r_{v}}{\sigma_{S D}^{2}}$ and $Z_{v}^{s c}=0$. Then, for $v \in \mathcal{V}_{s c}$, we have

$$
m_{v, c}^{s c}=Z_{v}^{s c}+\sum_{c^{\prime} \neq c} m_{c^{\prime}, v}^{s c}+f_{c}\left(m_{v}^{c c \rightarrow s c}, p_{e}\right)
$$

therefore,

$$
m_{v}^{c c \rightarrow s c}=Z_{v}^{c c}+\sum_{c^{\prime}} m_{c^{\prime}, v}^{c c}
$$

represents the messages transferred from the variable nodes $v$ of the channel decoder to the variable nodes of the source decoder. As previously described, and for $v \in \mathcal{V}_{s c}$, the source-relay link can be noisy, and the sequence c decoded by the relay node to obtain $\hat{\mathbf{s}}$ is damaged by errors with a bit error probability $p_{e}$. Indeed, to recover the signal $\mathbf{s}$, the proposed decoder applies the LLRs-based updating function $f_{c}$ according to equations (3) and (4) described above.

Thereafter, the messages for the $v \in \mathcal{V}_{c c}^{r e d}$ are given by

$$
m_{v, c}^{c c}=Z_{v}^{c c}+\sum_{c^{\prime} \neq c} m_{c^{\prime}, v}^{c c}
$$

We notice that the messages $m_{c^{\prime}, v}^{c c}$ and $m_{c^{\prime}, v}^{s c}$ are initially set to zero.

Second, the messages between the check nodes $c$ and the variable nodes $v$ for $c \in \mathcal{C}_{c c}$ are given by

$$
m_{c, v}^{c c}=2 \tanh ^{-1}\left(\prod_{v^{\prime} \neq v} \tanh \left(\frac{m_{v^{\prime}, c}^{c c}}{2}\right)\right)
$$

while for $c \in \mathcal{C}_{s c}$, the messages are:

$$
m_{c, v}^{s c}=2 \tanh ^{-1}\left(\tanh \left(\frac{Z_{c}^{s c}}{2}\right) \prod_{v^{\prime} \neq v} \tanh \left(\frac{m_{v^{\prime}, c}^{s c}}{2}\right)\right)
$$

where $Z_{c}^{s c}=\frac{2 r_{c}}{\sigma_{R D}^{2}}$ are the initial channel LLRs corresponds to noisy vector $\mathbf{b}$ received by the destination node from the relay. After a given number of iterations of the described joint decoder, the source symbols are estimated by

$$
\operatorname{LLR}(\mathbf{s})=Z_{v}^{s c}+\sum_{c} m_{c, v}^{s c}
$$




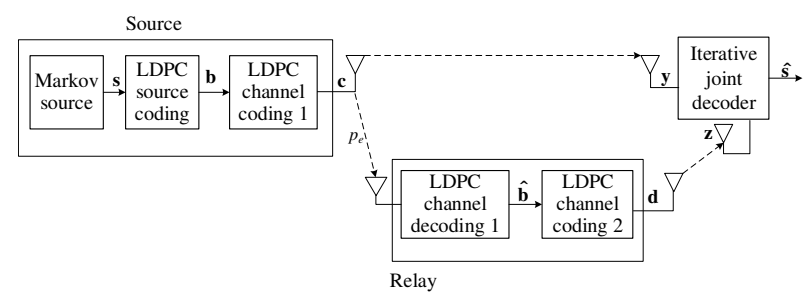

(a) System model for Source Channel Coding Strategy 3 (SCCS3)

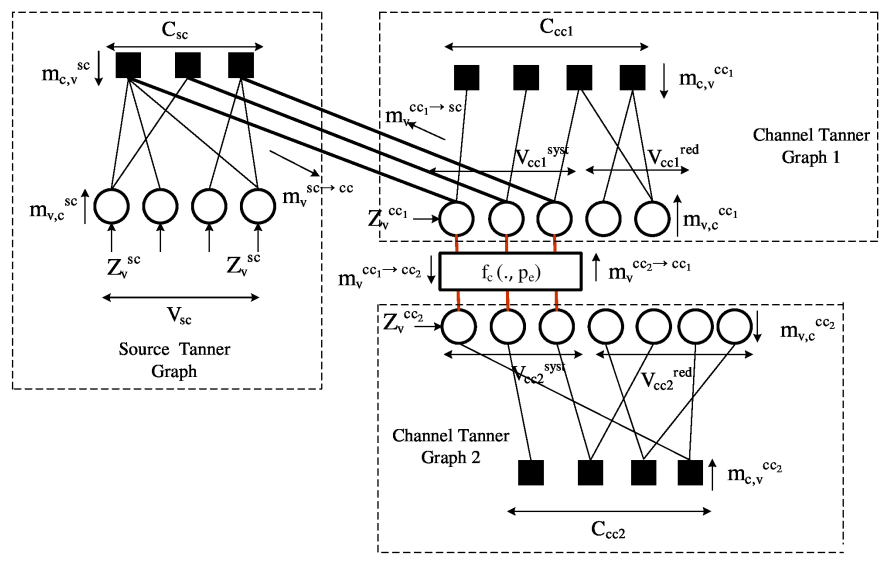

(b) Network Tanner graph and message transfer for SCCS3

Fig. 3: The SCCS3 for the cooperative system and the corresponding Joint Tanner graph decoder, exploiting source-relay correlation.

\section{SCC Strategy 3 (SCCS3)}

The main idea of the SCC Strategy 3 is to generalize the first proposed SCC Strategy 1 by making the source-relay link more reliable, using an LDPC source encoder followed by a first LDPC channel code at the source node, as shown in Fig. 3 (a). At the relay node, we apply a second LDPC channel code. During the first phase, at the source node, we use a double LDPC code for JSC coding. The first code compresses the data of the Markov source $\mathbf{s}$ using equation (1) to obtain a sequence b. Then, we protect this compressed sequence with an LDPC channel encoder according to

$$
\mathbf{c}=\mathbf{G}_{c c_{1}}^{T} \times \mathbf{b}
$$

to obtain an encoded sequence $\mathbf{c}$ with $m_{1}$ bits length. $\mathbf{G}_{c c_{1}}$ is the generator matrix of the first LDPC channel code. This encoded sequence is forwarded to both the relay and the receiver nodes. At the second phase, the relay node performs an LDPC channel decoding on the recovered sequence to estimate $\hat{\mathbf{b}}$. In addition, the decoded sequence is encoded by another LDPC channel code as

$$
\mathbf{d}=\mathbf{G}_{c c_{2}}^{T} \times \hat{\mathbf{b}}
$$

to generate the signal $\mathbf{d}$ of $m_{2}$ bits, which is transmitted to the destination over AWGN channel by means of BPSK modulation. $\mathbf{G}_{c c_{2}}$ is the generator matrix of the second LDPC channel code.

At the receiver, a joint decoder is applied by exchanging messages between variable and check nodes of the elementary decoders. This joint decoder can be described by three Tanner graphs, as shown in Fig. 3 (b) (we keep the same notations as in the previous sections). First, we describe the messages of the full joint Tanner graph decoder between the $v$ nodes and the $c$ nodes. For $v \in \mathcal{V}_{s c}$, each $v$ node informs about its LLR to the connected $c$ node as

$$
m_{v, c}^{s c}=Z_{v}^{s c}+\sum_{c^{\prime} \neq c} m_{c^{\prime}, v}^{s c}
$$

For $v \in \mathcal{V}_{c c_{1}}^{s y s t}$, on the side of the first LDPC channel decoder (channel Tanner graph 1), we have

$$
m_{v, c}^{c c_{1}}=Z_{v}^{c c_{1}}+\sum_{c^{\prime} \neq c} m_{c^{\prime}, v}^{c c c_{1}}+f_{c}\left(m_{v}^{c c c_{2} \rightarrow c c_{1}}, p_{e}\right)+m_{v}^{s c \rightarrow c c_{1}}
$$

where $Z_{v}^{c c_{1}}=\frac{2 r_{v}}{\sigma_{S D}^{2}}$ represents noisy version of the codeword $\mathbf{c}, m_{v}^{c c_{2} \rightarrow c c_{1}}$ represents the LLRs between the variable nodes from the second channel decoder graph to the first channel decoder graph, and $m_{v}^{s c \rightarrow c c_{1}}$ are the LLRs sent by the check nodes of the source decoder graph to the variable nodes of the channel decoder graph 1. Also, for $v \in \mathcal{V}_{c c_{1}}^{s y s}$, the messages passed from the variable node $v$ of the channel decoder graph 1 to the check node $c$ of the source decoder graph are given by:

$$
m_{v}^{c c_{1} \rightarrow s c}=Z_{v}^{c c_{1}}+\sum_{c^{\prime}} m_{c^{\prime}, v}^{c c_{1}}
$$


As mentioned above, the source-relay link is assumed to be noisy with an error probability $p_{e}$. The relay node observes a corrupted version of the codeword $\mathbf{c}$ which has been decoded to obtain $\hat{\mathbf{b}}$. The aim of the joint decoder is to compute $\mathbf{b}$ based on $\operatorname{LLR}(\hat{\mathbf{b}})$ using $f_{c}$ function for $v \in \mathcal{V}_{c c_{1}}^{s y s t}$. Then, for $v \in \mathcal{V}_{c c_{1}}^{r e d}$, we have

$$
m_{v, c}^{c c_{1}}=Z_{v}^{c c_{1}}+\sum_{c^{\prime} \neq c} m_{c^{\prime}, v}^{c c_{1}}
$$

For the second channel decoder (channel Tanner graph 2), the messages between variable and check nodes are described as follows. For $v \in \mathcal{V}_{\mathrm{Cc}_{2}}^{\text {syst }}$,

$$
m_{v, c}^{c c_{2}}=Z_{v}^{c c_{2}}+\sum_{c^{\prime} \neq c} m_{c^{\prime}, v}^{c c_{2}}+f_{c}\left(m_{v}^{c c_{1} \rightarrow c c_{2}}, p_{e}\right)
$$

where $m_{v}^{c c_{1} \rightarrow c c_{2}}$ are the messages passed between the variable nodes from the channel decoder 1 to the channel decoder 2 . After that, for $v \in \mathcal{V}_{c c_{2}}^{r e d}$,

$$
m_{v, c}^{c c_{2}}=Z_{v}^{c c_{2}}+\sum_{c^{\prime} \neq c} m_{c^{\prime}, v}^{c c c_{2}}
$$

with $Z_{v}^{c c_{2}}=\frac{2 r_{v}}{\sigma_{R D}^{2}}$ the initial channel LLR of the noisy encoded vector d. We note that the messages are initially set to $m_{c^{\prime}, v}^{c c_{1}}=0, m_{c^{\prime}, v}^{s c}=0, m_{v}^{s c \rightarrow c c_{1}}=0$, and $m_{c^{\prime}, v}^{c c_{2}}=0$.

Second, we describe the messages between the check nodes $c$ and the variable nodes $v$, which are given by the following expressions. For $c \in \mathcal{C}_{s c}$, we have,

$$
m_{c, v}^{s c}=2 \tanh ^{-1}\left(\prod_{v^{\prime} \neq v} \tanh \left(\frac{m_{v^{\prime}, c}^{s c}}{2}\right) \times \tanh \left(\frac{m_{v}^{c c_{1} \rightarrow s c}}{2}\right)\right)
$$

and

$$
m_{v}^{s c \rightarrow c c_{1}}=2 \tanh ^{-1}\left(\prod_{v^{\prime}} \tanh \left(\frac{m_{v^{\prime}, c}^{s c}}{2}\right)\right)
$$

Furthermore, for $c \in \mathcal{C}_{c c_{1}}$, the messages are described as

$$
m_{c, v}^{c c_{1}}=2 \tanh ^{-1}\left(\prod_{v^{\prime} \neq v} \tanh \left(\frac{m_{v^{\prime}, c}^{c c_{1}}}{2}\right)\right)
$$

For the second channel decoder, the LLRs between the check and the variable nodes for $c \in \mathcal{C}_{c c_{2}}$ are given by

$$
m_{c, v}^{c c_{2}}=2 \tanh ^{-1}\left(\prod_{v^{\prime} \neq v} \tanh \left(\frac{m_{v^{\prime}, c}^{c c_{2}}}{2}\right)\right)
$$

After a fixed number of iterations of the decoder, the information sequence $\hat{\mathbf{s}}$ is estimated based on LLR(s) such as

$$
\operatorname{LLR}(\mathbf{s})=Z_{v}^{s c}+\sum_{c} m_{c, v}^{s c}
$$

\section{Simulation Results}

In this section, we study the performance of the three proposed strategies with an equivalent overall rate. The source correlated data are modeled with a two-states Markov process, having the transition probabilities $\alpha=\beta=0.07$. Hence, the source entropy rate is $H(S)=0.3659$. We investigate the performance of the SCCSs even with errors in the source-relay link, and with three relay-position scenarios (Scenario A, Scenario B, Scenario C) as described in Fig. 4. we also notice that this work consider only regular LDPC codes for the source and the channel encoders. In the considered scenario, the relay node $R$ can be located closer to the destination node $D$ (scenario A) or to the source node $S$ (scenario B), or the three nodes maintain the same distance with each other (scenario C). We define by $G_{R D}=\left(\frac{d_{S D}}{d_{R D}}\right)^{n}$ the geometric gain s of the link between the nodes $R$ and $D$, with respect to the source-destination link distance, where $d_{S D}$ and $d_{R D}$ denote the distance from the source $S$ to $D$ and from the relay node $R$ to $D$, respectively. The path-loss exponent $n$ is assumed to be equal to 3.52 [36]. We note that the geometric gain of the source-destination link is normalized to 1. The SNRs of each scenario can be computed as the following

1) In scenario A, the $\mathrm{SNRs}$ of the relay-destination and the source-relay links are, $S N R_{R D}=S N R_{S D}+21.19 \mathrm{~dB}$ $\left(G_{R D}=\left(\frac{d_{S D}}{d_{R D}}\right)^{n}=\left(\frac{d}{d / 4}\right)^{3.52}=21.19 \mathrm{~dB}\right)$ and $S N R_{S R}=S N R_{S D}+4.4 \mathrm{~dB}\left(G_{S R}=\left(\frac{d_{S D}}{d_{S R}}\right)^{n}=\left(\frac{d}{3 d / 4}\right)^{3.52}=4.4 \mathrm{~dB}\right)$.

2) In scenario B, we have $S N R_{R D}=S N R_{S D}+4.4 \mathrm{~dB}\left(G_{R D}=\left(\frac{d_{S D}}{d_{R D}}\right)^{n}=\left(\frac{d}{3 d / 4}\right)^{3.52}=4.4 \mathrm{~dB}\right)$, and $S N R_{S R}=$ $S N R_{S D}+21.19 \mathrm{~dB}\left(G_{S R}=\left(\frac{d_{S D}}{d_{S R}}\right)^{n}=\left(\frac{d}{d / 4}\right)^{3.52}=21.19 \mathrm{~dB}\right)$.

3) Scenario $C$ supposes that the SNR of the source-destination link is equal to the source-relay and the relay-destination links, where $S N R_{R D}=S N R_{S D}=S N R_{S R}$. 

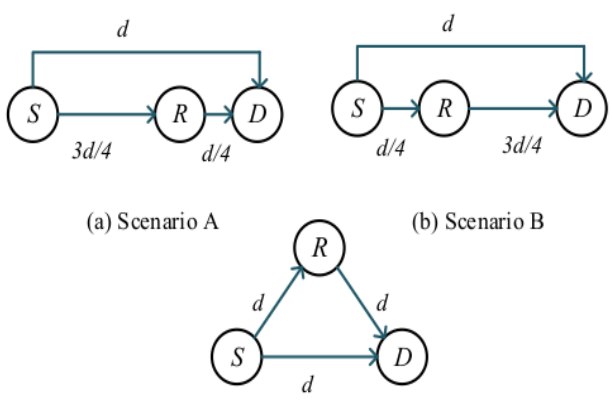

(c) Scenario C

Fig. 4: Three different positions scenarios of the cooperative communication system. $S, R$ and $D$ denotes respectively source, relay, and destination.

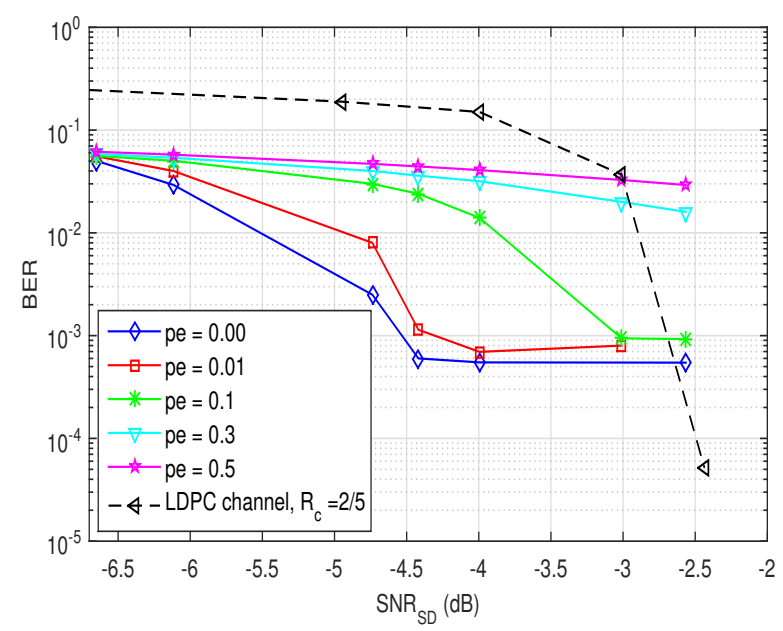

Fig. 5: BER performance for equivalent rates LDPC channel coding and SCC Strategy 1 with scenario A and different $p_{e}$ at the relay node, where $R_{s c}=1 / 2$ and $R_{c c}=1 / 4$.

\section{A. Performance analysis of the SCC Strategy 1 (SCCS1)}

1) SCC Strategy 1 using JSCC LDPC codes and a noisy source-relay link: In this section, we study the SCC Strategy 1 performance with regular LDPC source and LDPC channel codes distributed between the source and the relay nodes, respectively. We compare the performance of the proposed relay system with different positions of the relay node (Scenario A, Scenario B), and with noisy source-relay link, to an equivalent rate standard LDPC channel code.

The first regular LDPC code compresses the information source of $n=1800$ bits with a compression rate $R_{s c}=\frac{1}{2}$, and degrees defined by $\left(d_{v}=3, d_{c}=6\right)$. The second code protects the compressed data with a channel coding rate $R_{c c}=\frac{1}{4}$ and constant degrees $(3,4)$, which means an overall rate of $R=\frac{2}{5}$. For the reference LDPC channel code, we consider a regular code with an equivalent rate $R_{c}=\frac{2}{5}$. At the joint decoder, we apply 100 iterations of the iterative algorithm presented previously. In Fig. 5 and Fig. 6, we plot the BER as a function of the $S N R_{S D}$ for scenario A and scenario B, respectively. First, we can see that the performance of the SCC Strategy 1 with scenario A where the relay node is closer to the destination than the source node, is better than the LDPC channel coding in the waterfall region with different error probabilities $p_{e}$. We provide an improvement of about $2.6 \mathrm{~dB}$ with the error-free case for a BER equal to $10^{-2}$.

Through scenario A and scenario B, we can see that with the same error probability $p_{e}=0.01$ at the relay node, we almost keep the same performance. Moreover, we can remark that with $p_{e}=0.1$, the SCC Strategy 1 remains robust against observation errors occurred at the relay, due to the updating function $f_{c}$ which takes into account such errors in the source-relay link. Indeed, with $p_{e}=0.1$, we just loose $0.76 \mathrm{~dB}$ for a BER $=10^{-2}$ with scenario $\mathrm{B}$. We note that with high error probabilities as $p_{e}=0.3$ and $p_{e}=0.5$, the performance of the SCC Strategy 1 in both scenario A and B is damaged in the waterfall as in the error floor regions, since the $f_{c}$ function will almost disable any information transfer between the component decoders. The use of the LDPC channel coding at the source node could improve the reliability of the source-relay link, which will be considered in the following SCCS2. 


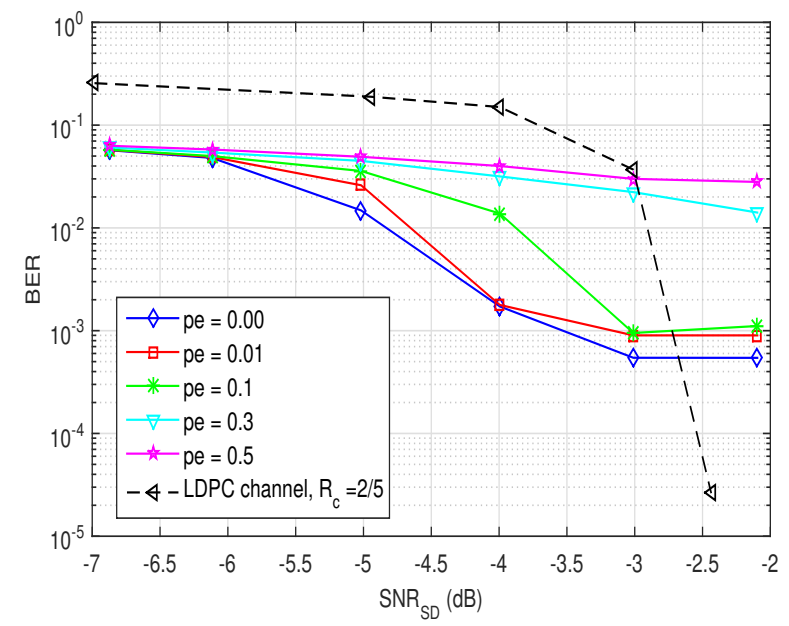

Fig. 6: BER performance for equivalent rates LDPC channel coding and SCC Strategy 1 with scenario B and different $p_{e}$, where $R_{s c}=1 / 2$ and $R_{c c}=1 / 4$.

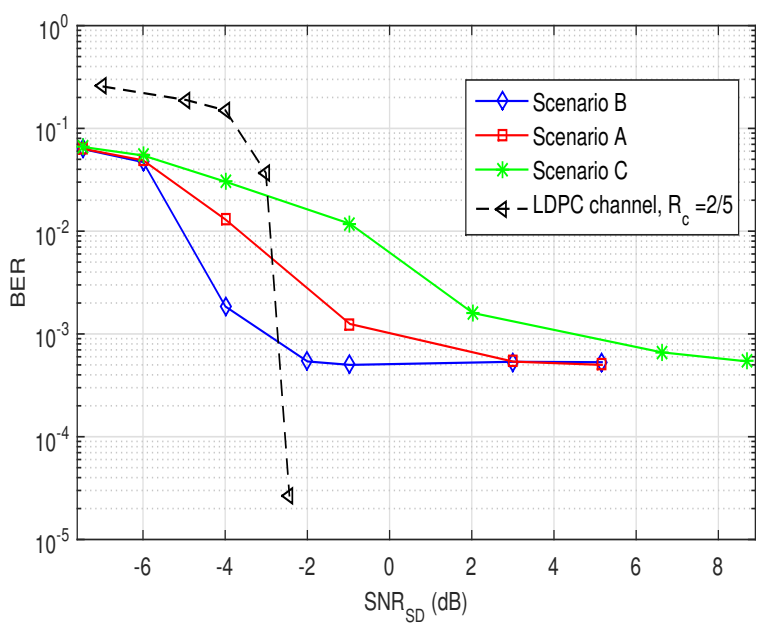

Fig. 7: Performance comparison of three relay positions, taken into account to $p_{e}$ between the $S-R$ link for the proposed Source Channel Coding Strategy 1 (SCCS1) where $R_{s c}=1 / 2$ and $R_{c c}=1 / 4$.

2) BER performance comparison of the proposed SCC Strategy 1 with three relay locations: We indicate that the sourcerelay link is corrupted by an error probability $p_{e}$, which depends on $S N R_{S R}$. To analyze and compare the system performance, Fig. 7 presents the BER performance of three relay positions where the error probability $p_{e}$ is taken into consideration by computing the $S N R_{S R}$ for each scenario.

In this case, we remark that the performance of the SCC Strategy 1 is better in the waterfall region with scenario B, where the relay node is closer to the source node than the destination, than the two other scenarios (A and $\mathrm{C}$ ). We obtain a gain of about $3 \mathrm{~dB}$ for a $\mathrm{BER}=10^{-3}$ with respect to scenario $\mathrm{A}$, and a gain of about $6 \mathrm{~dB}$ compared to scenario $\mathrm{C}$ for a $\mathrm{BER}=2 \cdot 10^{-3}$. In the error floor region, we keep the same values around $10^{-4}$, because the three scenarios have the same compression rate $R_{s c}=\frac{1}{2}$, and the same source characteristics.

\section{B. Performance analysis of the SCC Strategy 2 (SCCS2)}

In this paragraph, we study the performance of the SCC Strategy 2 with distributed JSCC LDPC regular codes, where the LDPC channel coding is done at the source node, and the LDPC compression operation is done at the relay node, with two relay locations (scenario A and scenario B). The proposed system is also compared to an equivalent rate, standard LDPC channel code. We consider $R_{c c}=\frac{1}{2}$ and $R_{s c}=\frac{1}{2}$ with $(3,6)$ degrees, which gives an overall transmission rate of $\frac{2}{5}$.

We plot the BER performance as a function of $S N R_{S D}$ for both scenario A and scenario B in Fig. 8. First, we notice that a slight improvement in terms of BER system performance in scenario A compared to scenario B is obtained. In addition, we 


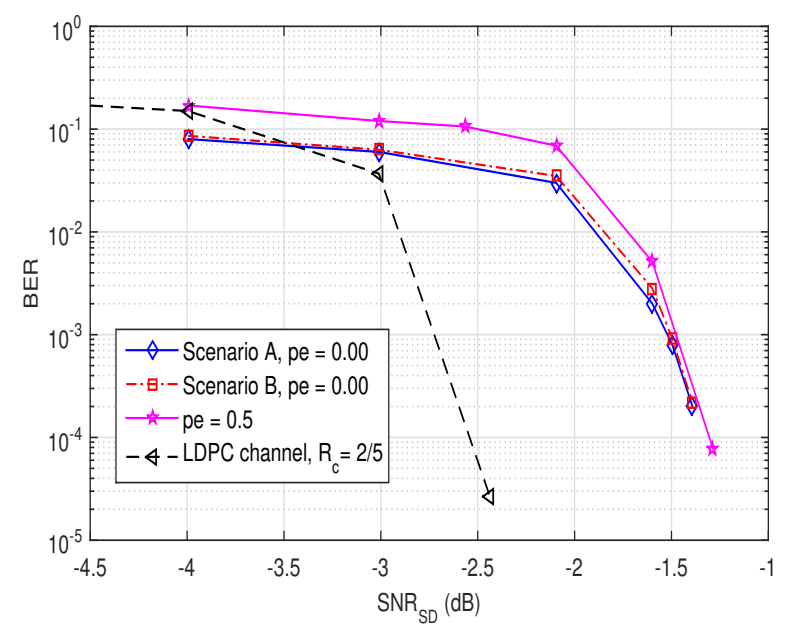

Fig. 8: BER performance for equivalent rates LDPC channel coding and SCC Strategy 2 with scenario A and scenario B and $p_{e}=0.5$ at the relay node, where $R_{s c}=1 / 2$ and $R_{c c}=1 / 2$.

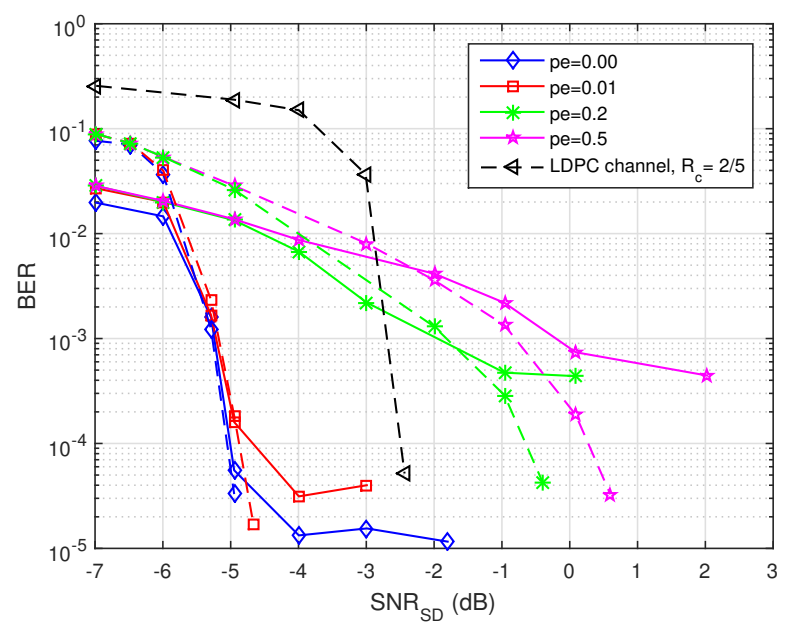

Fig. 9: BER performance for equivalent rates LDPC channel coding and SCC Strategy 3 with scenario B and different $p_{e}$, where $R_{s c}=1 / 2, R_{c c_{1}}=1 / 2$ and $R_{c c_{2}}=1 / 3$.

do not observe any error floor. Second, in the case of noisy source-relay link with error probability $p_{e}=0.5$, we observe a slight performance degradation of about $0.3 \mathrm{~dB}$ for a BER equal to $10^{-2}$ with scenario A. However, we remark that the LDPC channel code setup that takes into account the information sent by the source and the relay nodes, resulting an equivalent rate $R_{c}=\frac{2}{5}$, is better in terms of BER than the SCCS2 with a gain of about $1 \mathrm{~dB}$ in different scenarios of the relay position. Therefore, the idea of applying an LDPC source code at the relay node for the second SCCS2 is not reliable.

We conclude that the use of an LDPC source coding operation at the relay node for a cooperative network provides no significant improvements for the system performance. Indeed, allocating more bits derived from the relay to the source by reducing the channel coding rate applied at the source will be more efficient.

\section{Performance analysis of the SCC Strategy 3 (SCCS3)}

1) SCCS3 using JSCC LDPC codes and a noisy source-relay link: For the proposed SCC Strategy 3, we assume the use of a regular double source-channel LDPC code at the source node, while at the relay node, we apply another LDPC channel code. First, we analyze the performance of the SCC Strategy 3 with scenario B, where the relay is closer to the source than the destination, and we assume that the wireless link between the source and the relay is noisy.

In Fig. 9, we show the BER as a function of $S N R_{S D}$ for scenario B. The SCC Strategy 3 consists of three regular LDPC codes with rates $R_{s c}=\frac{1}{2}, R_{c c_{1}}=\frac{1}{2}$ and $R_{c c_{2}}=\frac{1}{3}$ (for the latter the degrees are defined by $(2,3)$ ) for the source, the channel coding 1 and the channel coding 2 , respectively. The overall rate is $\frac{2}{5}$. As in the previous sections, the reference LDPC channel 


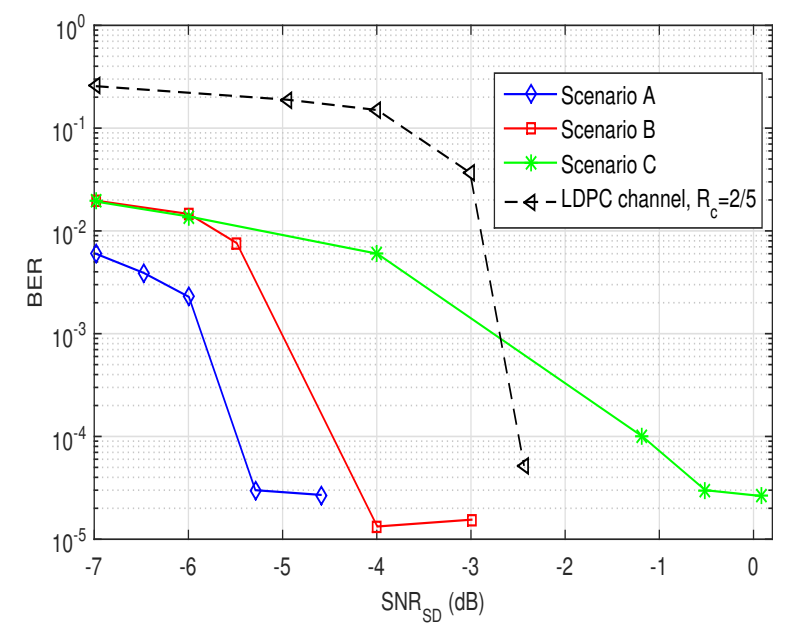

Fig. 10: Performance comparison of three relay positions, taken into account to $p_{e}$ between the source-relay link for SCC Strategy 3, where $R_{s c}=1 / 2, R_{c c_{1}}=1 / 2$ and $R_{c c_{2}}=1 / 3$.

code keeps the same equivalent rate of $\frac{2}{5}$. We observe that the performance of the SCC Strategy 3 with scenario B is better than the LDPC channel code in the waterfall region. We obtain a gain of about $4 \mathrm{~dB}$ for a BER=2.10 ${ }^{-2}$ with $p_{e}=0.00$. Then, in the case of a noisy source-relay link with different $p_{e}$, we notice that for relatively low error probabilities such as $p_{e}=0.01$, we keep almost the same performance as the case of error-free source-relay link.

In the same figure, we provide the asymptotic analysis of the BP algorithm for the used regular LDPC codes, using the mutual information measure based on EXIT charts over AWGN channel. The proposed decoder for SCCS3 is composed of three LDPC decoders, that exchange extrinsic messages between the variables and the check nodes in each Tanner graph, to estimate the source information based on the source variable nodes LLRs defined in eq. (36). We denote by $x_{i}^{s c}$ the extrinsic information at the output of the variable nodes of the LDPC source decoder, and $y_{i}^{s c}$ the a priori information at the input of the variable nodes of the LDPC source decoder. $x_{i}^{c c 1}, x_{i}^{c c 2}$ are, respectively, the extrinsic information at the output of the variable nodes of the LDPC channel decoder 1 and the LDPC channel decoder 2. $y_{i}^{c c 1}, y_{i}^{c c 2}$ are, respectively, the a priori information at the input of the variable nodes of the LDPC channel decoder 1 and the LDPC channel decoder 2. Also, we denote $V$, and $U$, the variable-to-check LLRs messages and the check-to-variable LLRs messages [2], [37]. $x_{i}^{s c}$ LLRs are given by

$$
x_{i}^{s c}=Z_{i}^{s c}+\left(d_{v}-1\right) \times y_{i}^{s c}
$$

where $y_{i}^{s c}=J^{-1}(I(X ; U))$, and $I(X ; U)$ is the mutual information between the received sequence $X$ and $U$ previously defined. $J(L)$ is defined by

$$
J(L)=I(X ; L)=1-\mathbf{E}\left[\log _{2}\left(1+e^{-L}\right)\right]
$$

So, the EXIT charts functions of the variable nodes of the source decoder are evaluated by

$$
\begin{aligned}
x^{s c}= & I(X ; V)=I\left(X ; U_{0}, U_{1}, \ldots, U_{d_{v}-1}\right) \\
& =J\left(J^{-1}\left(Z_{v}^{s c}\right)+\left(d_{v}-1\right) J^{-1}\left(y^{s c}\right)\right)
\end{aligned}
$$

where $x^{s c}$ is the average extrinsic information at the output of the variable nodes of the LDPC source decoder. $Z_{v}^{s c}$ is the average of $Z_{i}^{s c}$, and $y^{s c}=I(X ; U)=I\left(X ; V_{1}, \ldots, V_{d_{c}-1}\right)$ is the average a priori information at the input of the variable node of the LDPC source decoder. We estimate the source bits, after a given number of iterations, by computing the BER, based on the LLRs output by the variable nodes of the source decoder. We finally calculate the bit-error probability as, $P_{e}=\left[\mu_{0} Q\left(x_{s c}^{\mu_{0}}\right)+\left(1-\mu_{0}\right) Q\left(x_{s c}^{\left(1-\mu_{0}\right)}\right)\right]$, where $Q($.$) is the Gaussian tail function.$

2) Performance comparison of the three relay locations for SCC Strategy 3: In this subsection, we keep the same SCC Strategy 3 presented above. As previously shown, we compute the $S N R_{S R}$ between source-relay link, and we deduce the corresponding $p_{e}$. Thus, in Fig. 10, we compare the performance of the three scenarios of the relay position. We can distinguish that the performance of the SCCS3 with scenario A where the relay node is closer to the destination than the source node is better than the scenario $\mathrm{B}$ and scenario $\mathrm{C}$. We observe a gain of about $0.85 \mathrm{~dB}$ for a $\mathrm{BER}=10^{-3}$ with the appearance of an error floor for $\mathrm{SNR}=-5.25 \mathrm{~dB}$ compared to scenario $\mathrm{B}$, and a gain equal to $3 \mathrm{~dB}$ for a $\mathrm{BER}=10^{-3}$ compared to scenario $\mathrm{C}$. 
TABLE I: Different configurations depends on the source code rate $R_{s c}$, and the channel code rates $R_{c c_{1}}$ and $R_{c c_{2}}$ for SCC Strategy 3 (SCCS3)

\begin{tabular}{|c|c|c|c|}
\hline Configurations & $\begin{array}{c}\text { Source coding } \\
\text { rate, } R_{s c}\end{array}$ & $\begin{array}{c}\text { Channel coding } \\
\text { rate } 1, R_{c c_{1}}\end{array}$ & $\begin{array}{c}\text { Channel coding } \\
\text { rate } 2, R_{c c_{2}}\end{array}$ \\
\hline Conf 1 & $1 / 2$ & $2 / 7$ & $2 / 3$ \\
\hline Conf 2 & $1 / 2$ & $1 / 3$ & $1 / 2$ \\
\hline Conf 3 & $1 / 2$ & $1 / 2$ & $1 / 3$ \\
\hline Conf 4 & $1 / 2$ & $3 / 4$ & $3 / 11$ \\
\hline Conf 5 & $5 / 18$ & $1 / 6$ & $1 / 3$ \\
\hline Conf 6 & $1 / 3$ & $2 / 9$ & $1 / 3$ \\
\hline Conf 7 & $5 / 9$ & $1 / 2$ & $2 / 5$ \\
\hline Conf 8 & $5 / 18$ & $1 / 3$ & $1 / 6$ \\
\hline Conf 9 & $1 / 3$ & $1 / 3$ & $2 / 9$ \\
\hline
\end{tabular}

According to the performance of the SCC Strategy 1 and the SCC Strategy 2 previously analyzed, with and without errors in the wireless link between the source and relay nodes, and with different location scenarios of the relay, we can remark that the performance of the SCC Strategy 3 in both waterfall and error floor regions is the best than the others proposed relay systems (SCCS1 and SCCS2). However, the system performance still depends on the source and channel codes rates.

3) Effect of the channel coding rates for the SCC Strategy 3 with scenario A and scenario B: The idea behind this study is to show the effect of the LDPC channel coding rate $1, R_{c c_{1}}$, and the LDPC channel coding rate $2, R_{c c_{2}}$ on the SCC Strategy 3 results.

We propose to study four configurations (Conf 1, Conf 2, Conf 3, and Conf 4) with the same overall transmission rate of $2 / 5$ and with the same compression rate, $R_{s c}=1 / 2$ as shown in Table. I. We note that simulations take into account the error probability $p_{e}$ occurring in the source-relay link.

Fig. 11 shows the BER performance as a function of $S N R_{S D}$ for the four proposed configurations with scenario A. We observe that the first configuration improves the waterfall region of the system in terms of BER with a lower protection capacity, $R_{c c_{1}}=\frac{2}{7}$ at the source node, and $R_{c c_{2}}=\frac{2}{3}$ at the relay node, providing an improvement of about $0.5 \mathrm{~dB}$ compared to the second configuration for a BER $=10^{-3}$. However, the second configuration protects less at the source node with $R_{c c_{1}}=\frac{1}{3}$, while $R_{c c_{2}}=\frac{1}{2}$ at the relay node, and shows a performance degradation in the waterfall region. Also, we observe that the configuration 4 protects less the data at the source node than the first and the second configurations with $R_{c c_{1}}=\frac{3}{4}$, which induces a loss in the BER performance. Indeed, we loose $0.8 \mathrm{~dB}$ for a BER $=10^{-4}$ compared to the third configuration. Moreover, we notice the same error floor for the four configurations, which is justified by the use of the same compression rate.

In Fig. 12, we propose to use the same configurations (Conf 1, Conf 2, Conf 3, and Conf 4) but, in the case of scenario B, where the relay is assumed to be closer to the source than the destination. We can see that the fourth configuration improves the waterfall region performance compared to the others, which makes more protection at the relay node with $R_{c c_{2}}=\frac{3}{11}$. Although, the third configuration protects less at the relay node with $R_{c c_{2}}=\frac{1}{3}$, thus decreases of the performance in the waterfall region with a gain equal to $0.3 \mathrm{~dB}$ for a $\mathrm{BER}=10^{-3}$. Compared to the other configurations, the performance of the first one is the worst with $R_{c c_{2}}=\frac{2}{3}$ at the relay node.

We conclude that the waterfall region depends mainly on the channel coding rate $1\left(R_{c c_{1}}\right)$ for the SCCS3 with scenario A, since the relay is closer to the $D$ than the $S$, and using lower correction capacity at the source node, involves improvement in the waterfall region. Besides, in the case of scenario $\mathrm{B}$, the waterfall region depends on the channel coding rate $2\left(R_{c c_{2}}\right)$, since the relay is closer to the $S$ than the $D$, with a lower channel code rate at the relay node.

4) Effect of the source coding rates for the SCC Strategy 3 with scenario A: In this paragraph, we study the effect of the source coding rates on the SCC Strategy 3. As shown in Table. I, we propose different configurations (Conf 5, Conf 6, Conf 7) with the same overall rate, and we take lower correction capacity at the source node, with $R_{c c_{1}}$, according to the conclusion of the previous section, since we study the case of scenario A. We note that the error probability $p_{e}$ in the wireless source-relay link is always taken into account in simulations. In Fig. 13, we can see that the fifth configuration making more compression $\left(R_{s c}=\frac{5}{18}\right)$, provides an error floor at almost $S N R_{S D}=-6 \mathrm{~dB}$. However, the sixth configuration that compresses less with $R_{s c}=\frac{1}{3}$, generates a lower reduced error floor. Configuration 7, which compresses less, induces an even lower error floor with a BER $=10^{-5}$.

5) Effect of the source coding rates for the SCC Strategy 3 with scenario B: We keep the same idea to study the effect the source coding rate, and we focus on the behavior of the SCC Strategy 3 with scenario B (Conf 7, Conf 8, Conf 9). We observe in Fig. 14 that the configuration 8 that compresses more $\left(R_{s c}=\frac{5}{18}\right)$ and protects more the data at the relay node $\left(R_{c c_{2}}=\frac{1}{6}\right)$ than the two other codes, provides the best performance for the waterfall region at a cost of higher error floors. Indeed, an error floor appears at $S N R_{S D}=-5.5 \mathrm{~dB}$ and an improvement in terms of BER in the waterfall region is observed compared to 


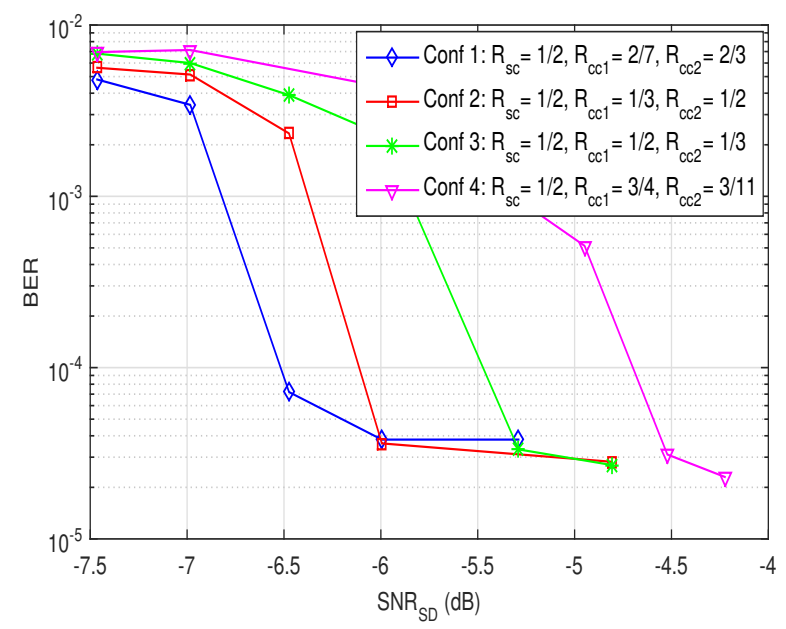

Fig. 11: BER performance of the SCC Strategy 3 with scenario A and different channel coding rates.

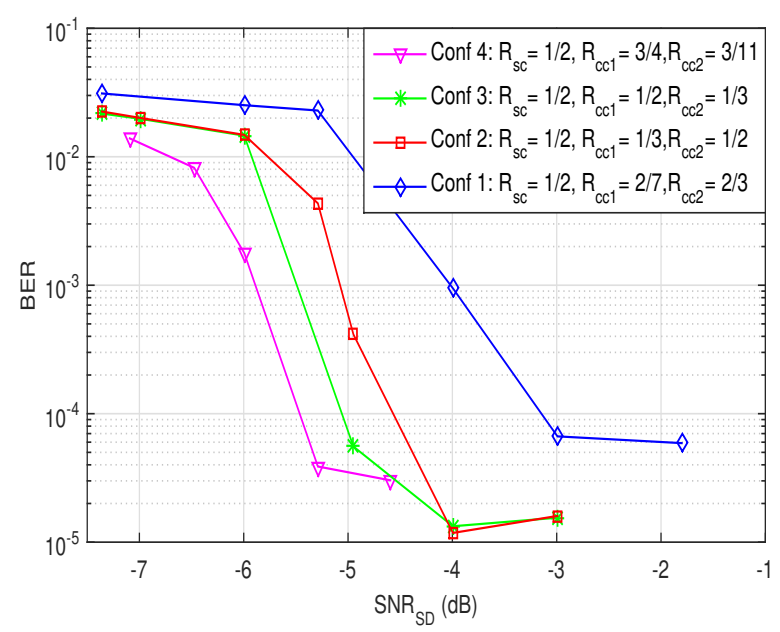

Fig. 12: BER performance of the SCC Strategy 3 with scenario B and different channel coding rates.

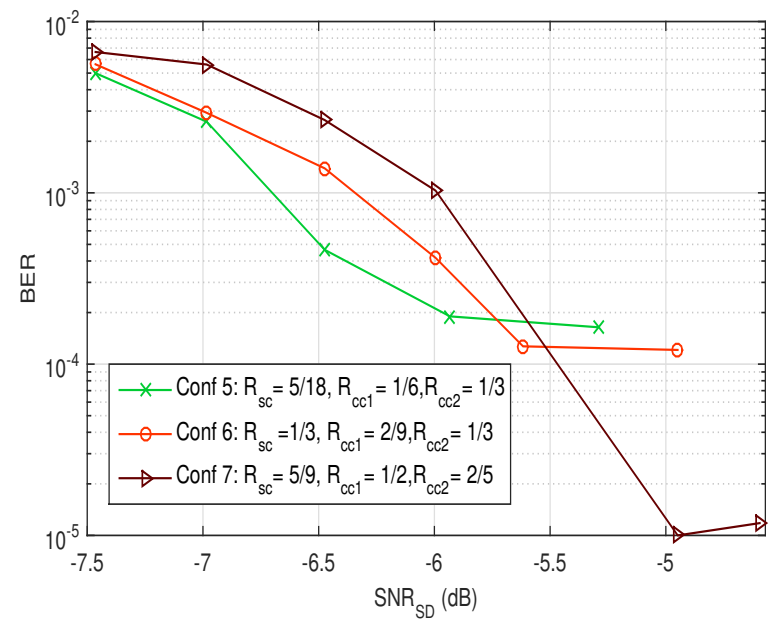

Fig. 13: BER performance of the SCC Strategy 3 (SCCS3) with scenario A and different source coding rates. 


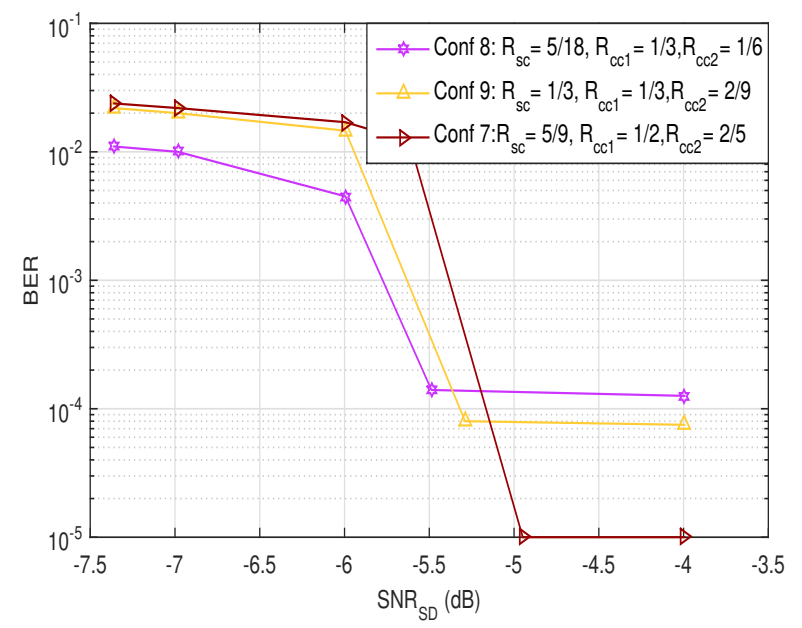

Fig. 14: BER performance of the SCCS3 with scenario B and different source coding rates.

the others configurations. Nevertheless, the seventh configuration compresses less $\left(R_{s c}=\frac{5}{9}\right)$, which induces lower error floor with a BER $=10^{-5}$.

Through Fig. 13 and Fig. 14 for scenario A and scenario B, respectively, we conclude that the error floor region depends on the compression rates and the use of lower compression rates achieves higher error floor with a higher SNRs. The waterfall performance mainly depends on the relay position setup and the channel coding rates allocation.

\section{CONCLUSIONS}

In this paper, we presented three Source Channel Coding Strategies (SCCSs) for cooperative networks with distributed source and channel coding operations based on JSCC LDPC codes. In the first SCC Strategy 1, we distributed the source and the channel coding operations between the source and the relay nodes, respectively. On the opposite, in the second SCC Strategy 2, we proposed an LDPC channel coding at the source node and an LDPC source coding at the relay node. For the SCC Strategy 3, the proposed system model is composed of a JSCC LDPC code applied at the source node and an LDPC channel coding operation applied at the relay node. Then, we developed for each corresponding SCCS an iterative joint decoder based on a modified BP algorithm that takes into consideration the source-relay possible error probabilities. We studied the SCCSs performance with different relay location scenarios, when the source-relay link is noisy and we compared the performance to an LDPC channel code with an equivalent rate. We conclude that the performance of the relay system depends on the coding strategy and the relay position, but also on the rate allocation. Therefore, the optimization of the rate allocation as a function of SNR will be considered as the future work.

\section{ACKNOWLEDGEMENT}

This research is in part supported by the Young Researcher Project COMMUN (18PJEC11-01) funded by the Ministry of Higher Education and Scientific Research of Tunisia.

\section{REFERENCES}

[1] C. E. Shannon, "A mathematical theory of communication,” Bell System Technical Journal, vol. 27, pp. 379-423, 623-656, 1948.

[2] M. Fresia, F. Pérez-Cruz, and H. V. Poor, "Optimized concatenated LDPC codes for joint source-channel coding," IEEE International Symposium Information Theory, (ISIT), pp. 2131-2135, Jun. 2009.

[3] M. Fresia, F. Pérez-Cruz, H. V. Poor, and S. Verdu, "Joint source and channel coding," IEEE Sig. Proc. Mag., vol. 27, no. 6, pp. 104-113, Nov. 2010. https://doi.org/10.1109/MSP.2010.938080.

[4] C. Chen, L. Wang, and S. Liu, "The Design of Protograph LDPC Codes as Source Codes in a JSCC System," IEEE Communications Letters, vol. 22, no. 4, pp. 672-675, 2018.

[5] G. Han, J. Jiang, M. Guizani, and J. J. C. Rodrigues, "Green routing protocols for wireless multimedia sensor networks," IEEE Wireless Communications, vol. 23, no. 6, pp. 140-146, 2016.

[6] k. P. Seng, and L. M. Ang, "A big data layered architecture and functional units for the multimedia Internet of Things," IEEE Transactions on Multi-Scale Computing Systems, vol. 4, no. 4, pp. 500-512, 2018.

[7] L. Pu, Z. Wu, A. Bilgin, M. W. Marcellin, and B. Vasic, "LDPC-based iterative joint source-channel decoding for JPEG2000," IEEE Transactions on Image Processing, vol. 16, no. 2, pp. 577-581, 2007.

[8] A. Zribi, R. Pyndiah, S. Zaibi, F. Guilloud, and A. Bouallègue, "Low-complexity soft decoding of Huffman codes and iterative joint source channel decoding," IEEE Transactions on Communications, vol. 60, no. 6, pp. 1669-1679, 2012.

[9] J. Liu, G. Tu, C. Zhang, and Y. Yang, "Joint source and channel decoding for variable length encoded turbo codes," EURASIP Journal on Advances in Signal Processing, vol. 2008, no. 1, 2007. 
[10] J. Hagenauer, and R. Bauer, "The turbo principle in joint source channel decoding of variable length codes," Information Theory Workshop. Proceedings, pp. 33-35, 2001.

[11] A. Guyader, E. Fabre, C. Guillemot, and M. Robert, "Joint source-channel turbo decoding of entropy-coded sources," IEEE Journal on Selected Areas in Communications, vol. 19, no. 9, pp. 1680-1696, 2001.

[12] M. Izhar, N. Fisal, X. Zhoo, K. Anwar, and T. Matsumoto, "Utilization of 2-D Markov source correlation using block turbo codes ", Proc. of the 7th Intl. Symposium on Turbo Codes and Iterative Information Processing 2012, pp. 56--60, 2012.

[13] Y. Zhao, R. Adve, and T. J. Lim, "Improving amplify-and-forward relay networks: optimal power allocation versus selection," IEEE International Symposium on Information Theory, pp. 1234-1238, 2006.

[14] B. Zhao, and M. C. Valenti, "Distributed turbo coded diversity for relay channel," Electronics letters, vol. 39, no. 10, pp. 786-787, 2003.

[15] R. Blasco-Serrano, R. Thobaben, and M. Skoglund, "Compress-and-forward relaying based on symbol-wise joint source-channel coding," IEEE International Conf. on Communications, pp. 1-5, 2010.

[16] S. X. Ng, Y. Li, and L. Hanzo, "Distributed turbo trellis coded modulation for cooperative communications," International Conference on Communications (ICC), June 2009.

[17] Y. Fang, S. C. Liew, and T. Wang, "Design of distributed protograph LDPC codes for multi-relay coded-cooperative networks," IEEE Transactions on Wireless Communications, vol. 16, no. 11, pp. 7235-7251, 2017.

[18] T. J. Richardson, and R. Urbanke, "The capacity of low-density parity-check codes under message-passing decoding," IEEE Transactions on information theory, vol. 47, no. 2, pp. 599-618, 2001.

[19] A. Chakrabarti, A. De Baynast, A. Sabharwal, and B. Aazhang, "Low density parity check codes for the relay channel," IEEE journal on selected areas in communications, vol. 25, no. 2, pp. 280-291, 2007.

[20] J. Hu, and T. M. Duman, "Low density parity check codes over wireless relay channels," IEEE Transactions on Wireless Communications, vol. 6, no. 9, 2007.

[21] Y. Fang, G. Bi, and Y. L. Guan "Design and analysis of root-protograph LDPC codes for non-ergodic block-fading channels," IEEE Transactions on Wireless Communications, vol. 14, no. 2, pp. 738-749, 2015.

[22] Y. Fang, P. Chen, G. Cai, F. C. Lau, S. C. Liew, and G. Han "Outage-Limit-Approaching Channel Coding for Future Wireless Communications: Root-Protograph Low-Density Parity-Check Codes," IEEE Vehicular Technology Magazine, vol. 14, no. 2, pp. 85-93, 2019.

[23] D. Duyck, J. J. Boutros, and M. Moeneclaey, "Low-density graph codes for coded cooperation on slow fading relay channels," IEEE Transactions on Information Theory, vol. 57, no. 7, pp. 4202-4218, 2011.

[24] J. P. Cances, and V. Meghdadi, "Optimized low density parity check codes designs for half duplex relay channels," IEEE Transactions on Wireless Communications, vol. 8, no. 7, 2009.

[25] G. Cai, Y. Fang, G. Han, J. Xu, and G. Chen, "Design and analysis of relay-selection strategies for two-way relay network-coded DCSK systems," IEEE Transactions on Vehicular Technology, vol. 67, no. 2, pp. 1258-1271, 2017.

[26] X. Zhou, M. Cheng, k. Anwar, and T. Matsumoto, "Distributed joint source-channel coding for relay systems exploiting source-relay correlation and source memory," EURASIP Journal on Wireless Communications and Networking, pp. 132-136, Aug. 2012. https://doi.org/10.1186/1687-1499-2012-260.

[27] A. D. Murugan, P. K. Gopala, and H. El Gamal, "Correlated sources over wireless channels: Cooperative source-channel coding," IEEE Journal on Selected Areas in Communications, vol. 22, no. 6, pp. 988-998, 2004.

[28] k. Anwar, and T. Matsumoto, "Accumulator-assisted distributed turbo codes for relay systems exploiting source-relay correlation," IEEE Communications Letters, vol. 16, no. 7, pp. 1114-1117, 2012.

[29] L. Xu, H. Wu, J. He, and L. Wang, "Unequal error protection for radiography image transmission using protograph double LDPC codes," IEEE Wireless Telecommunications Symposium, pp. 1-5, 2013.

[30] Q. Chen, L. Wang, S. Hong, and Z. Xiong, "Performance improvement of JSCC scheme through redesigning channel code," IEEE Communications Letters, vol. 20, no.6, pp. 1088-1091, 2016.

[31] M. Ben Abdessalem, A. Zribi, T. Matsumoto, and A. Bouallègue, "LDPC-based Joint Source-Channel-Network Coding for the Multiple Access Relay Channel," International Conference on Wireless Networks and Mobile Communications (WINCOM), October 2018.

[32] Y. Fang, G. Bi, Y. L. Guan, and F. C. Lau, "A survey on protograph LDPC codes and their applications," IEEE Communications Surveys and Tutorials, vol. 14, no. 4, pp. 1989-2016, 2015.

[33] S. Hong, Q. Chen, and L. Wang, "Performance analysis and optimisation for edge connection of JSCC system based on double protograph LDPC codes," IET Communications, vol. 12, no. 2, pp. 214-219, 2017

[34] C. Chen, L. Wang, and F. C. Lau, "Joint optimization of protograph LDPC code pair for joint source and channel coding," IEEE Transactions on Communications, vol. 66, no. 8 pp. 3255-3267, 2018.

[35] L. Deng, Z. Shi, O. Li, and J. Ji, "Joint Coding and Adaptive Image Transmission Scheme Based on DP-LDPC Codes for IoT Scenarios," IEEE Access, vol. 7, pp. 18437-18449, 2019.

[36] R. Youssef, and A. G. i Amat, "Distributed serially concatenated codes for multi-source cooperative relay networks," IEEE transactions on wireless communications, vol. 10, no. 1, pp. 253-263, 2011.

[37] E. Sharon, A. Ashikhmin, and S. Litsyn, "Analysis of low-density parity-check codes based on EXIT functions," IEEE Transactions on Communications, vol. 54, no. 8, pp. 1407-1414, 2006. 